
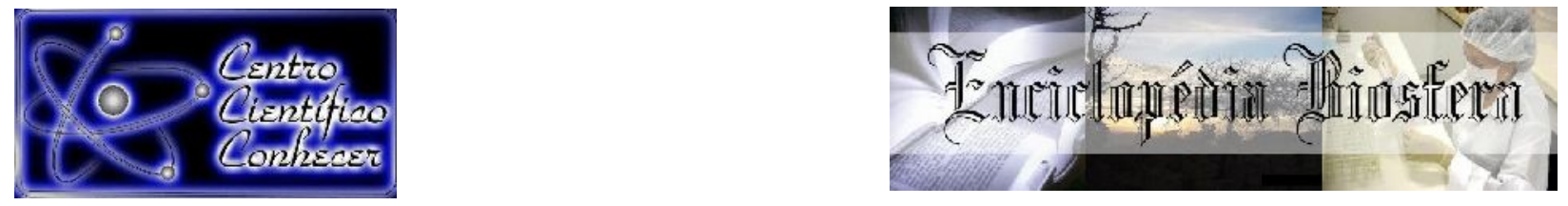

\title{
USO DA ESCALA DE DEPRESSÃO GERIÁTRICA NO CONTEXTO DA ATENÇÃO PRIMÁRIA À SAÚDE
}

Luiz Henrique Batista Monteiro ${ }^{1}$, Juliana Nogueira Pontes Nobre ${ }^{2}$, Paulo Filipe de Mello $^{3}$, Rosana Passos Cambraia ${ }^{4}$ Renata Aline de Andrade ${ }^{5}$

1- Enfermeiro. Mestrando do Programa de Pós-Graduação em Saúde, Sociedade e Ambiente, Universidade Federal dos Vales do Jequitinhonha e Mucuri

(UFVJM). Diamantina, Minas Gerais, Brasil.(luizhbmonteiro@gmail.com)

2- Educadora Física. Mestre em Saúde, Soc. e Ambiente pela Universidade Federal dos Vales do Jequitinhonha e Mucuri (UFVJM). Diamantina, Minas Gerais, Brasil 3- $\quad$ Enfermeiro. Mestre em Saúde, Soc. e Ambiente pela Universidade Federal dos Vales do Jequitinhonha e Mucuri (UFVJM). Diamantina, Minas Gerais, Brasil 4- $\quad$ Psicobióloga. Depto de Farmácia, Faculdade de Ciências Biológicas e da Saúde, Universidade Federal dos Vales do Jequitinhonha e Mucuri. Diamantina, Minas Gerais, Brasil

5- $\quad$ Farmacêutica-bioquímica. Depto de Farmácia, Faculdade de Ciências Biológicas e da Saúde, Universidade Federal dos Vales do Jequitinhonha e Mucuri. Diamantina, Minas Gerais, Brasil

Recebido em: 22/09/2018 - Aprovado em: 23/11/2018 - Publicado em: 03/12/2018 DOI: 10.18677/EnciBio_2018B106

\section{RESUMO}

Objetivo: Conhecer o perfil dos trabalhos científicos e avaliar o uso da EDG-15 no rastreamento de sintomas depressivos em idosos que vivem na comunidade, e os fatores de riscos associados a estes sintomas. Método: Revisão integrativa da literatura nas bases LILACS e SciELO utilizando os seguintes descritores: 'atenção primária à saúde', depressão', 'envelhecimento', 'idosos'. Considerou-se o período de 2013 a 2017 para a seleção dos artigos publicados em português, espanhol ou inglês. Foi realizada busca manual (hand search) nos artigos selecionados. Resultados: Doze artigos atenderam aos critérios de inclusão, destes, 5 (41,7\%) foram selecionados na base LILACS e 7 (58,8\%) no SciELO. E a prevalência de sintomas depressivos encontrada nesta revisão variou de $18,0 \%$ a $88,8 \%$. Destacaram-se como fatores de riscos para a presença de sintomas depressivos as variáveis sociodemográficas, condições de saúde, hábitos de vida e avaliação negativa do estado de saúde. Conclusão: O tema desta revisão é de suma importância, porém existe escassez de produção cientifica. Contudo, assim, a Escala de Depressão Geriátrica é um instrumento empregado em larga escala para avaliação da presença de sintomas depressivos na APS. Presume-se que este trabalho poderá subsidiar decisões a respeito do investimento público, visando o fortalecimento da APS, com ênfase na saúde mental da população idosa.

PALAVRAS-CHAVE: Atenção Primária à Saúde. Depressão. Idoso. 


\title{
THE USE OF THE GERIATRIC DEPRESSION SCALE IN THE CONTEXT OF PRIMARY HEALTH CARE: INTEGRATIVE REVIEW
}

\begin{abstract}
Objective: To know the profile of the scientific work and to evaluate the use of EDG15 in the screening of depressive symptoms in elderly people living in the community, and the risk factors associated with these symptoms.Method: Integrative literature review in the LILACS and SciELO databases using the following descriptors: 'primary health care', 'depression', 'aging', 'elderly'. It was considered the period from 2013 to 2017 for the selection of articles published in Portuguese, Spanish or English. Hand search was performed on selected articles. Results: Twelve articles met the inclusion criteria, of which, $5(41.7 \%)$ were selected in the LILACS database and $7(58.8 \%)$ in the SciELO. And the prevalence of depressive symptoms found in this review ranged from $18.0 \%$ to $88.8 \%$. The sociodemographic variables, health conditions, life habits and negative evaluation of health status were highlighted as risk factors for the presence of depressive symptoms. Conclusion: The theme of this review is of great importance, but there is a shortage of scientific production. However, Geriatric Depression Scale is a widely used instrument for assessing the presence of depressive symptoms in PHC. It is assumed that this work may subsidize decisions regarding public investment, aiming to strengthen $\mathrm{PHC}$, with an emphasis on the mental health of the elderly population.
\end{abstract}

KEYWORDS: Aged. Depression. Primary Health Care.

\section{INTRODUÇÃO}

Estima-se que a depressão seja responsável por $4,3 \%$ da carga global de doenças em nível mundial (WHO, 2013). Além disto, é considerada como a principal causa de incapacidade no mundo, associada a elevadas taxas de morbidade e mortalidade e agravos crônicos não transmissíveis (WHO, 2012; SILVA et al., 2014; FENG et al., 2014). Estudos internacionais indicam que a prevalência de depressão em idosos residentes em comunidades oscila de 16,5\% a 38,0\% (KANG et al., 2013; FENG et al., 2014; KAUP et al., 2016; SANDERS et al., 2016).

Em relação a depressão geriátrica, esta configura-se como o transtorno mental mais frequente entre os idosos. Entre estes a depressão pode potencializar a probabilidade para o aparecimento e evolução de incapacidades funcionais, compromete a qualidade de vida, eleva os gastos orçamentários dos serviços públicos, além de caracterizar fator de risco para a ideação suicida, e consequentemente as tentativas e o suicídio consumado (CAVALCANTE et al., 2013; WHO, 2015; JUNG et al., 2017). Neste seguimento, o uso de escalas reconhecidas mundialmente colabora para a detecção dos casos de depressão geriátrica, posto que são de fácil e rápida aplicação, simples compreensão, e baixo custo operacional (ALVARENGA et al., 2010; NOGUEIRA et al., 2014; BRETANHA et al., 2015).

Internacionalmente e no Brasil, uma das escalas utilizadas para avaliação de sintomas depressivos em populações idosas é a "Geriatric Depression Scale" (GDS), criada por Yesavage em 1983, inicialmente com 30 itens que correlacionam o diagnóstico depressivo. Esta escala foi traduzida para o português e adaptada para aplicação no Brasil por Almeida e Almeida (1999), sendo utilizadas as versões de 30 (REBELLO et al., 2011), de 10 itens (HELLWIG et al., 2016) e a versão de 15 itens. Vale salientar que esta última é a mais utilizada, e em diferentes contextos clínicos (TESTON et al., 2014; COHEN et al., 2015; TAVARES et al., 2016; NASCIMENTO 
et al., 2017; GUTHS et al., 2017; MELO et al., 2017; CONFORTIN et al., 2017; FLUETTI et al., 2018; RIBEIRO et al., 2018).

É fundamental notabilizar que são poucas as evidências acerca da identificação da depressão geriátrica mediante as ações dos profissionais de saúde. Reconhece-se que estes sintomas não são detectados efetivamente, e assim sendo não são tratados (LIMA et al., 2009; HOFFMANN et al., 2010). Nesse sentido, esta revisão de literatura objetivou conhecer o perfil dos trabalhos científicos e avaliar o uso da EDG-15 no rastreamento de sintomas depressivos em idosos que vivem na comunidade, e os fatores de riscos associados a estes sintomas.

\section{ETAPAS DA REVISÃO}

O presente trabalho trata-se de uma Revisão Integrativa de Literatura (RI). A $\mathrm{RI}$ é uma metodologia de pesquisa que objetiva analisar e sistematizar as publicações científicas relacionadas a determinado tema. A RI consiste na associação e seguimento das seis etapas elencadas a seguir: identificação do tema, seleção da questão norteadora, delineamento dos critérios de inclusão e exclusão dos artigos científicos a serem analisados, determinação das informações que são extraídas da produção cientifica selecionada, avaliação dos estudos incluídos, interpretação dos resultados e apresentação dos resultados oriundos da revisão (MENDES et al., 2008). Nesse sentido, a RI é uma importante ferramenta utilizada na área da saúde, uma vez que tem a finalidade de sintetizar estudos científicos a respeito de determinado tema e direcionar a prática embasada no conhecimento científico (SOUZA et al., 2010).

Foram incluídos artigos científicos publicados na íntegra e originais, oriundos de pesquisas científicas desenvolvidas no Brasil, que utilizaram a EDG-15 para a triagem de sintomas depressivos entre idosos. O período entre os anos de 2013 a 2017 foi delimitado para a seleção dos artigos publicados em português, espanhol ou inglês. Os estudos obrigatoriamente deveriam versar acerca do objetivo de investigar a presença de sintomas depressivos entre idosos que vivem na comunidade, por meio da aplicação da EDG-15. Os estudos repetidos foram excluídos.

Após a leitura dos artigos as informações de interesse foram extraídas, fundamentadas em um instrumento validado (URSI; GALVAO, 2006). As informações extraídas relacionam-se a autoria, título do artigo, base de dados, delineamento do estudo, síntese dos resultados e das conclusões.

Para a busca da produção científica selecionaram-se os descritores presentes nos Descritores em Ciências da Saúde (DeCS) para indexação dos estudos: "envelhecimento", "depressão", "idosos", "atenção primária a saúde". As bases de dados utilizadas foram: Scientific Electronic Library Online (SciELO) e Literatura Latino-Americana e do Caribe em Ciências da Saúde (LILACS).

No tocante a pesquisa dos artigos, foi realizada a busca manual (hand search), com o intuito de localizar nas referências selecionadas dos bancos de dados, artigos referenciados com o propósito de explorar outras produções científicas (GALVÃO et al., 2010). A seleção dos artigos foi concretizada entre os meses de março a junho de 2018, em conformidade com a figura 1. 


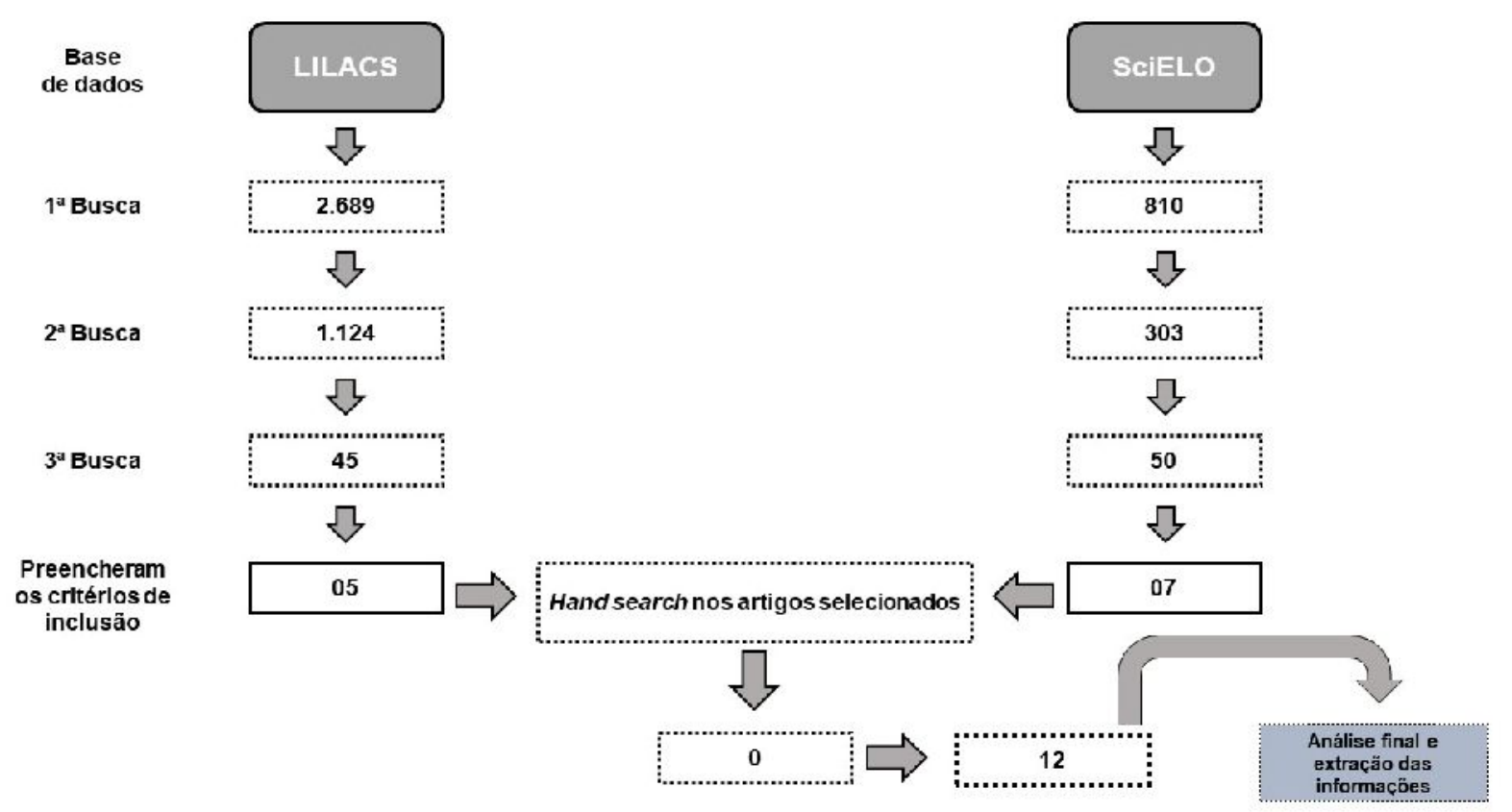

FIGURA 1: Processo de seleção dos artigos da amostra, 2013-2017.

\section{APRESENTAÇÃO E DESENVOLVIMENTO DA REVISÃO}

Esta RI compreende a análise de 12 artigos, em consonância aos critérios de inclusão e exclusão determinados pelos autores. Destes, $5(41,7 \%)$ foram selecionados da LILACS e $7(58,8 \%)$ da SciELO. A prevalência de sintomas depressivos na população idosa identificada neste estudo variou de $18,0 \%$ a $88,8 \%$. O quadro 1 enumera os achados substanciais desta revisão.

QUADRO 1: Síntese dos artigos que utilizaram a EDG-15, 2013-2017.

\begin{tabular}{|c|c|c|c|c|c|}
\hline Título & Autores & $\begin{array}{c}\text { Ano/Cidade- } \\
\text { UF/ } \\
\text { Base de } \\
\text { dados/ } \\
\text { Amostra }\end{array}$ & $\begin{array}{l}\text { Delineamento } \\
\text { do estudo/ } \\
\text { Instrumentos }\end{array}$ & Resultados & $\begin{array}{l}\text { Síntese das } \\
\text { conclusões }\end{array}$ \\
\hline $\begin{array}{l}\text { Depressão em } \\
\text { idosos } \\
\text { hipertensos e } \\
\text { diabéticos no } \\
\text { contexto da } \\
\text { atenção } \\
\text { primária em } \\
\text { saúde }\end{array}$ & MADEIRA et al. & $\begin{array}{l}\text { 2013/ São Luís- } \\
\text { MA/ LILACS/ } 66\end{array}$ & $\begin{array}{c}\text { Transversal/ } \\
\text { EDG-15 e } \\
\text { questionário } \\
\text { sociodemográfico. }\end{array}$ & $\begin{array}{c}\text { - 31,8\% identificados } \\
\text { com sintomas } \\
\text { depressivos. } 27,3 \% \\
\text { com sintomas leves e } \\
4,5 \% \text { com sintomas } \\
\text { graves. } \\
\text { - } 50 \% \text { dos idosos } \\
\text { identificados com } \\
\text { sintomas depressivos } \\
\text { tinham } 75 \text { anos ou } \\
\text { mais. }\end{array}$ & $\begin{array}{c}\text { - Alta } \\
\text { prevalência de } \\
\text { sintomas } \\
\text { depressivos } \\
\text { em idosos que } \\
\text { tinham } \\
\text { Hipertensão } \\
\text { Arterial e } \\
\text { Diabetes } \\
\text { mellitus. }\end{array}$ \\
\hline $\begin{array}{c}\text { Perfil } \\
\text { sociodemográfic } \\
\text { o e de saúde de } \\
\text { idosos com } \\
\text { sintomas } \\
\text { depressivos }\end{array}$ & SOUZA et al. & $\begin{array}{l}\text { 2013/ JEQUIÉ- } \\
\text { BA/ LILACS/ } 125\end{array}$ & $\begin{array}{c}\text { Transversal/ } \\
\text { EDG-15 e } \\
\text { questionários com } \\
\text { questões } \\
\text { sociodemográfica } \\
\text { s, patologias } \\
\text { referidas e } \\
\text { autopercepção do } \\
\text { estado de saúde. }\end{array}$ & $\begin{array}{c}\text { - 88,8\% identificados } \\
\text { com sintomas } \\
\text { depressivos. } \\
\text { - Mais propensos a } \\
\text { sintomas depressivos, } \\
\text { mulheres, idosos na } \\
\text { faixa etária de } 60 \text { a } 69 \\
\text { anos, viver com } \\
\text { companheiro, }\end{array}$ & $\begin{array}{l}\text {-Variáveis } \\
\text { sociodemográf } \\
\text { icas e de } \\
\text { saúde } \\
\text { representaram } \\
\text { fatores de } \\
\text { risco para } \\
\text { sintomatologia } \\
\text { depressiva. }\end{array}$ \\
\hline
\end{tabular}




\begin{tabular}{|c|c|c|c|c|c|}
\hline & & & & $\begin{array}{c}\text { analfabetismo e } \\
\text { presença de alguma } \\
\text { patologia. }\end{array}$ & \\
\hline $\begin{array}{l}\text { Depressão em } \\
\text { idosos na } \\
\text { Estratégia } \\
\text { Saúde da } \\
\text { Família: uma } \\
\text { contribuição } \\
\text { para a atenção } \\
\text { primária }\end{array}$ & $\begin{array}{l}\text { MAGALHAES } \\
\text { et al. }\end{array}$ & $\begin{array}{l}\text { 2016/ Teresina-PI/ } \\
\text { LILACS/ } 241\end{array}$ & $\begin{array}{c}\text { Transversal/ } \\
\text { EDG-15 e } \\
\text { questões } \\
\text { sociodemográfica } \\
\text { s. }\end{array}$ & $\begin{array}{c}\text { - 29,1\% identificados } \\
\text { com sintomas } \\
\text { depressivos. Destes } \\
26,6 \% \text { com sintomas } \\
\text { leves, e } 2,5 \% \\
\text { sintomas graves. } \\
\text { - Os sintomas foram } \\
\text { mais prevalentes em } \\
\text { mulheres, } \\
\text { participantes com } \\
\text { idade de } 70 \text { a } 79 \text { anos, } \\
\text { idosos que viviam sem } \\
\text { companheiro, inativos } \\
\text { e analfabetos. }\end{array}$ & $\begin{array}{c}\text { - Significativa } \\
\text { prevalência de } \\
\text { sintomas } \\
\text { depressivos } \\
\text { nos idosos } \\
\text { participantes. }\end{array}$ \\
\hline $\begin{array}{c}\text { Sintomas } \\
\text { depressivos e } \\
\text { fatores } \\
\text { associados em } \\
\text { idosos na } \\
\text { atenção } \\
\text { primária à } \\
\text { saúde }\end{array}$ & HAJJAR et al. & $\begin{array}{c}\text { 2017/ Uberaba- } \\
\text { MG/ LILACS/ } 248\end{array}$ & $\begin{array}{l}\quad \text { Transversal/ } \\
\text { EDG-15/ } \\
\text { Questionário } \\
\text { sociodemográfico, } \\
\text { condições de } \\
\text { saúde e hábitos } \\
\text { de vida. }\end{array}$ & $\begin{array}{c}\text { - 32,7\% identificados } \\
\text { com sintomas } \\
\text { depressivos. Sendo, } \\
24,4 \% \text { sintomas leves } \\
\text { e 7,3\% sintomas } \\
\text { graves. } \\
\text { - Fatores de risco: não } \\
\text { praticar exercício } \\
\text { físico, maior número } \\
\text { de morbidades. }\end{array}$ & $\begin{array}{c}\text { - Poucos } \\
\text { idosos } \\
\text { identificados } \\
\text { com sintomas } \\
\text { depressivos } \\
\text { faziam uso de } \\
\text { antidepressivo. }\end{array}$ \\
\hline $\begin{array}{l}\text { Prevalência de } \\
\text { sintomas de } \\
\text { depressão em } \\
\text { idosos } \\
\text { assistidos pela } \\
\text { Estratégia de } \\
\text { Saúde da } \\
\text { Família }\end{array}$ & SOUSA et al. & $\begin{array}{l}\text { 2017/ Cajazeiras- } \\
\text { PB/ LILACS/ } 153\end{array}$ & $\begin{array}{l}\text { Transversal/ } \\
\text { EDG-15/ } \\
\text { Questionário } \\
\text { sociodemográfico, } \\
\text { condições de } \\
\text { saúde e religião. }\end{array}$ & $\begin{array}{c}\text { - 50,9\% identificados } \\
\text { com sintomas } \\
\text { depressivos. } \\
\text { - Fatores de risco: } \\
\text { sexo femininos, } \\
\text { divorciados, sem } \\
\text { religião e com doença } \\
\text { crônica. }\end{array}$ & $\begin{array}{c}\text { A prevalência } \\
\text { de sintomas } \\
\text { depressivos } \\
\text { entre os } \\
\text { idosos foi } \\
\text { semelhante } \\
\text { aos resultados } \\
\text { de outros } \\
\text { estudos. }\end{array}$ \\
\hline $\begin{array}{c}\text { Fatores } \\
\text { associados aos } \\
\text { sintomas } \\
\text { depressivos em } \\
\text { idosos: } \\
\text { EpiFloripa }\end{array}$ & BORGES et al. & $\begin{array}{c}2013 / \\
\text { Florianópolis-SC/ } \\
\text { SciELO/ } 1656\end{array}$ & $\begin{array}{l}\text { Transversal/ } \\
\text { EDG-15 } \\
\text { e questionário } \\
\text { estruturado } \\
\text { (sociodemografi- } \\
\text { cas, pratica de } \\
\text { religião, grupo de } \\
\text { convivência, uso } \\
\text { da internet e } \\
\text { telefone, } \\
\text { avaliação da } \\
\text { saúde e dor) } \\
\text { Miniexame do } \\
\text { Estado Mental, } \\
\text { Alcohol Use } \\
\text { Disorders } \\
\text { Identification Test } \\
\text { (AUDIT), } \\
\text { International } \\
\text { Physical Activity } \\
\text { Questionnaire } \\
\text { (IPAQ), } \\
\text { Avaliação do } \\
\text { Índice de Massa }\end{array}$ & $\begin{array}{l}\text { - 23,9\% identificados } \\
\text { com sintomas } \\
\text { depressivos. } \\
\text { - Fatores de risco: } \\
\text { baixa escolaridade, } \\
\text { situação econômica } \\
\text { pior quando } \\
\text { comparada aos } 50 \\
\text { anos, déficit cognitivo, } \\
\text { percepção de saúde } \\
\text { regular e ruim, } \\
\text { dependência funcional } \\
\text { e dor crônica. } \\
\text { - Fatores de proteção: } \\
\text { Grupo etário de } 70 \\
\text { a79 anos, prática de } \\
\text { atividade física e de } \\
\text { lazer, participação em } \\
\text { grupos de convivência } \\
\text { e religiosos e vida } \\
\text { sexual ativa. }\end{array}$ & $\begin{array}{c}\text { O } \\
\text { rastreamento } \\
\text { de sintomas } \\
\text { depressivos } \\
\text { em idosos é } \\
\text { imprescindível. } \\
\text { E necessário a } \\
\text { criação de } \\
\text { políticas de } \\
\text { atenção à } \\
\text { saúde do } \\
\text { idoso na } \\
\text { cidade do } \\
\text { estudo. }\end{array}$ \\
\hline
\end{tabular}




\begin{tabular}{|c|c|c|c|c|c|}
\hline & & & $\begin{array}{c}\text { Corporal (IMC) } \\
\text { Atividades de } \\
\text { Vida Diária (AVD) } \\
\text { e Atividades } \\
\text { Instrumentais de } \\
\text { Vida Diária } \\
\text { (AIVD) }\end{array}$ & & \\
\hline $\begin{array}{c}\text { Rastreamento } \\
\text { de sintomas } \\
\text { depressivos em } \\
\text { idosos na } \\
\text { Estratégia } \\
\text { Saúde da } \\
\text { Família, Porto } \\
\text { Alegre. }\end{array}$ & $\begin{array}{l}\text { NOGUEIRA et } \\
\text { al. }\end{array}$ & $\begin{array}{c}\text { 2014/ Porto } \\
\text { Alegre-RS/ } \\
\text { SciELO/ } 585\end{array}$ & $\begin{array}{l}\text { Transversal/ } \\
\text { EDG-15 e } \\
\text { questionário com } \\
\text { perguntas sobre } \\
\text { os dados } \\
\text { sociodemográfico } \\
\text { s, religião e } \\
\text { avalição do } \\
\text { estado de saúde. }\end{array}$ & $\begin{array}{c}\text { - 30,6\% foram } \\
\text { identificados com } \\
\text { sintomas depressivos. } \\
\text { - Fatores de risco: } \\
\text { sexo feminino, baixa } \\
\text { escolaridade, } \\
\text { sobretudo } \\
\text { analfabetismo, } \\
\text { autopercepção de } \\
\text { saúde (regular e } \\
\text { ruim/péssima). }\end{array}$ & $\begin{array}{l}\text { É necessário } \\
\text { melhorar o } \\
\text { diagnóstico e } \\
\text { identificação } \\
\text { de depressão } \\
\text { em idosos. }\end{array}$ \\
\hline $\begin{array}{c}\text { Sintomas } \\
\text { depressivos em } \\
\text { idosos } \\
\text { residentes em } \\
\text { áreas de } \\
\text { abrangência das } \\
\text { Unidades } \\
\text { Básicas de } \\
\text { Saúde da zona } \\
\text { urbana de Bagé, } \\
\text { RS }\end{array}$ & $\begin{array}{l}\text { BRETANHA et } \\
\text { al. }\end{array}$ & $\begin{array}{c}\text { 2015/Bagé-RS/ } \\
\text { SciELO/ } 1593\end{array}$ & $\begin{array}{c}\text { Transversal/ } \\
\text { EDG-15 e } \\
\text { questionários com } \\
\text { informações } \\
\text { sociodemográfica } \\
\text { s, presença de } \\
\text { morbidades } \\
\text { avaliação da } \\
\text { saúde e } \\
\text { Atividades de } \\
\text { Vida Diária (AVD) } \\
\text { e Atividades } \\
\text { Instrumentais de } \\
\text { Vida Diária } \\
\text { (AIVD) }\end{array}$ & $\begin{array}{c}\text { - 18,0\% apresentaram } \\
\text { sintomas depressivos. } \\
\text { - Fatores de risco para } \\
\text { sintomas depressivos: } \\
\text { sexo feminino cor de } \\
\text { pele não branca, baixo } \\
\text { nível socioeconômico, } \\
\text { aposentados, com } \\
\text { histórico de problemas } \\
\text { cardíacos, idosos com } \\
\text { incapacidade e } \\
\text { dependência } \\
\text { funcional, avaliação } \\
\text { regular e ruim/ } \\
\text { péssima da saúde. } \\
\text { Fatores de proteção: } \\
\text { aposentado pelo } \\
\text { INSS. }\end{array}$ & $\begin{array}{c}\text { A prevalência } \\
\text { de sintomas } \\
\text { depressivos na } \\
\text { população } \\
\text { idosa, } \\
\text { impulsiona a } \\
\text { criação de } \\
\text { estratégias de } \\
\text { promoção da } \\
\text { saúde. }\end{array}$ \\
\hline $\begin{array}{l}\text { Prevalência de } \\
\text { sintomas } \\
\text { depressivos e } \\
\text { fatores } \\
\text { associados em } \\
\text { idosos no norte } \\
\text { de Minas } \\
\text { Gerais: um } \\
\text { estudo de base } \\
\text { populacional. }\end{array}$ & RAMOS et al. & $\begin{array}{c}\text { 2015/ Montes } \\
\text { Claros-MG/ } \\
\text { SciELO/ } 639\end{array}$ & $\begin{array}{c}\text { Transversal/ } \\
\text { EDG-15 e } \\
\text { questionário } \\
\text { estruturado com } \\
\text { questões } \\
\text { sociodemografica } \\
\text { s, estilo de vida, } \\
\text { histórico de } \\
\text { quedas, número } \\
\text { de morbidades, } \\
\text { presença de } \\
\text { cuidados, } \\
\text { avaliação da } \\
\text { saúde e } \\
\text { fraqilidade. }\end{array}$ & $\begin{array}{c}\text { - 27,5\% identificados } \\
\text { com sintomatologia } \\
\text { depressiva. } \\
\text { - Fatores de risco: } \\
\text { viver sem } \\
\text { companheiro, } \\
\text { analfabetismo, } \\
\text { avalição negativa da } \\
\text { saúde, uso de tabaco, } \\
\text { alto risco de quedas e } \\
\text { ser frágil }\end{array}$ & $\begin{array}{l}\text { Prevalência de } \\
\text { sintomatologia } \\
\text { alta, e } \\
\text { necessária } \\
\text { melhoria na } \\
\text { assistência } \\
\text { ofertada aos } \\
\text { idosos. }\end{array}$ \\
\hline $\begin{array}{l}\text { Depressão entre } \\
\text { idoso: um } \\
\text { estudo de base } \\
\text { populacional no } \\
\text { sul do Brasil }\end{array}$ & $\begin{array}{c}\text { GULLICH; } \\
\text { DURO; CESAR }\end{array}$ & $\begin{array}{l}\text { 2016/ Arroio } \\
\text { Trinta-SC/ } \\
\text { SciELO/ } 544\end{array}$ & $\begin{array}{c}\text { Transversal/ } \\
\text { EDG-15 e } \\
\text { instrumento com } \\
\text { questões } \\
\text { sociodemográfica } \\
\text { s, hábitos de vida, } \\
\text { prática religiosa, } \\
\text { atividades de } \\
\text { lazer, }\end{array}$ & $\begin{array}{l}\text { - 20,4\% identificados } \\
\text { com sintomas } \\
\text { depressivos. } \\
\text { - Fatores de risco: } \\
\text { sexo feminino, ser } \\
\text { solteiro, baixa renda, } \\
\text { ser fumante } \\
\text { hospitalizações nos } \\
\text { últimos } 12 \text { meses. }\end{array}$ & $\begin{array}{c}\text { A presença de } \\
\text { sintomas } \\
\text { depressivos } \\
\text { em idosos é } \\
\text { uma realidade. } \\
\text { Os resultados } \\
\text { são } \\
\text { semelhantes a } \\
\text { achados de }\end{array}$ \\
\hline
\end{tabular}




\begin{tabular}{|c|c|c|c|c|c|}
\hline & & & $\begin{array}{l}\text { hospitalização e } \\
\text { consultas médica. }\end{array}$ & $\begin{array}{l}\text { - Fatores de proteção: } \\
\text { participação em } \\
\text { eventos religiosos e } \\
\text { prática de atividade } \\
\text { física (última semana). }\end{array}$ & $\begin{array}{c}\text { investigações } \\
\text { realizadas em } \\
\text { grandes } \\
\text { centros } \\
\text { urbanos. }\end{array}$ \\
\hline $\begin{array}{l}\text { Active aging is } \\
\text { associated with } \\
\text { low prevalence } \\
\text { of depressive } \\
\text { symptoms } \\
\text { among Brazilian } \\
\text { older adults }\end{array}$ & GALLI et al. & $\begin{array}{c}2016 / \\
\text { Veranópolis-RS/ } \\
\text { SciELO/ } 1012\end{array}$ & $\begin{array}{c}\text { Cross-cut/ } \\
\text { GDS-15 } \\
\text { Structured } \\
\text { questionnaire } \\
\text { (sociodemographi } \\
\text { c information, } \\
\text { evaluation of } \\
\text { health status, } \\
\text { number of } \\
\text { morbidities, } \\
\text { number of } \\
\text { consultations last } \\
\text { month, } \\
\text { participation in } \\
\text { groups, work } \\
\text { situation, } \\
\text { interaction with } \\
\text { friends, manual } \\
\text { work, reading and } \\
\text { practicing } \\
\text { physical } \\
\text { activities). }\end{array}$ & $\begin{array}{c}\text { - } 36.6 \% \text { identified with } \\
\text { depressive symptoms. } \\
\text { - Risk factors: female } \\
\text { sex, } 75 \text { years of age or } \\
\text { older, low } \\
\text { socioeconomic level, } \\
\text { low educational level, } \\
\text { higher number of } \\
\text { consultations, } \\
\text { morbidities and poor } \\
\text { health evaluation. } \\
\text { - Protective factors: } \\
\text { work, physical activity, } \\
\text { perform manual work, } \\
\text { interest in reading and } \\
\text { talking with friends. }\end{array}$ & $\begin{array}{l}\text { Active aging is } \\
\text { important for } \\
\text { the mental } \\
\text { health of the } \\
\text { elderly. }\end{array}$ \\
\hline $\begin{array}{l}\text { Doenças } \\
\text { crônicas não } \\
\text { transmissíveis e } \\
\text { fatores } \\
\text { sociodemográfic } \\
\text { os associados a } \\
\text { sintomas de } \\
\text { depressão em } \\
\text { idosos. }\end{array}$ & SILVA et al. & $\begin{array}{c}\text { 2017/ Porto } \\
\text { Alegre-RS/ } \\
\text { SciELO/1391 }\end{array}$ & $\begin{array}{c}\text { Transversal/ } \\
\text { EDG-15 e } \\
\text { questionário } \\
\text { estruturado com } \\
\text { questões } \\
\text { sociodemográfica } \\
\text { s, diagnóstico de } \\
\text { doenças crônicas } \\
\text { não } \\
\text { transmissíveis. }\end{array}$ & $\begin{array}{l}\text { - Cerca de } 21 \% \text { foram } \\
\text { identificados com } \\
\text { sintomas depressivos. } \\
\text { - Fatores de risco: } \\
\text { sexo feminino, } \\
\text { doenças coronarianas, } \\
\text { insuficiência cardíaca, } \\
\text { acidente vascular } \\
\text { encefálico. } \\
\text { - Fatores de proteção: } \\
\text { escolaridade acima de } \\
8 \text { anos, ser viúvo e } \\
\text { viver com } \\
\text { companheiro. }\end{array}$ & $\begin{array}{l}\text { Há uma } \\
\text { possível } \\
\text { relação entre } \\
\text { depressão e } \\
\text { doenças } \\
\text { cardíacas e } \\
\text { comprometime } \\
\text { nto encefálico. } \\
\text { A escolaridade } \\
\text { é um fator de } \\
\text { proteção para } \\
\text { sintomas } \\
\text { depressivos. }\end{array}$ \\
\hline
\end{tabular}

De acordo com os critérios de inclusão, em relação ao ano de publicação dos estudos, 2013, 2016 e 2017, tiveram cada oito (25\%) de artigos analisados. $\mathrm{O}$ ano de 2014 teve um (8,3\%) e em 2015 foram dois artigos. Evidenciou-se que os artigos analisados $(58,3 \%)$ tinham como autor principal, ou coautores, profissionais de nível superior da área de enfermagem. Quanto ao idioma $11(91,7 \%)$ foram publicados na língua portuguesa do Brasil e um artigo (8,3\%) foi publicitado em inglês.

Em relação ao local de realização das pesquisas, seis $(50,0 \%)$ são advindas da região sul do Brasil, quatro (33,3\%) da região nordeste e duas (16,7\%) foram realizados na região sudeste. Observou-se predominância de investigações desenvolvidas no sul e sudeste com oito (66,7\%) artigos. Isso se deu em razão de que a região sudeste ocupa o primeiro lugar nas produções científicas em todas as áreas de conhecimento, sobretudo da saúde seguida da região sul (SANTOS et al., 2012; SIDONE et al., 2016).

No tocante ao delineamento do estudo, todos os artigos referem-se a pesquisas epidemiológicas, com abordagem transversal. O estudo transversal ou ENCICLOPÉDIA BIOSFERA, Centro Científico Conhecer - Goiânia, v. 15 n.28; p.1358 
seccional constitui-se em uma estratégia de pesquisa epidemiológica identificada pela observação direta e planejada de determinada quantidade de indivíduos em um único momento (KLEIN; BLOCK, 2009).

A EDG-15, conforme demonstrado nesta RI, é usada em larga escala no contexto da Atenção Primária a Saúde (APS). Em alguns estudos é evidenciada por esse instrumento, além da prevalência de sintomas depressivos, a diferenciação da prevalência de sintomas depressivos leves e graves. É relevante acentuar que idosos que apresentam pontuação na EDG-15 de zero a cinco apresentam ausência de sintomas depressivos. Já aqueles com pontuação na escala de seis a 10 indicam sintomas depressivos leves, e pontuação igual ou superior a 11 pontos sinalizam para a presença de sintomas graves (ALMEIDA; ALMEIDA, 1999).

De acordo com esta RI, existem fatores de risco para o desenvolvimento de sintomas depressivos em idosos. Em relação aos fatores sociodemográficos destacam-se: sexo feminino (MADEIRA et al., 2013; SOUZA et al., 2013; NOGUEIRA et al., 2014; BRETANHA et al., 2015; RAMOS et al., 2015; GALLI et al., 2016; GULLICH et al., 2016; MAGALHAES et al., 2016; SOUSA et al., 2017; SILVA et al., 2017), viver sem companheiro (SOUZA et al., 2013; RAMOS et al., 2015; GULLICH et al, 2016; MAGALHAES et al., 2016; SOUSA et al., 2017), baixo nível socioeconômico (BORGES et al., 2013; BRETANHA et al., 2015; GULLICH et al., 2016; GALLI et al., 2016) baixo nível educacional (principalmente analfabetismo) (SOUZA et al., 2013; BORGES et al., 2013; NOGUEIRA et al., 2014; RAMOS et al., 2015; GALLI et al., 2016), não ter religião (RAMOS et al., 2015; SOUSA et al., 2017).

Ser do sexo feminino é um fator de risco para o desenvolvimento de sintomas depressivos, em razão do isolamento social, arranjo familiar insatisfatório, sobrecarga das funções e pelas alterações hormonais (ANDRADE et al., 2006; NOGUEIRA et al., 2014; CHAN et al., 2012; GULLICH et al., 2016; MAGALHÃES et al., 2016).

A escolaridade foi reconhecida como fator de proteção para o desenvolvimento de sintomas depressivos, visto que a mesma maximiza o enfretamento da população frente aos eventos estressores (OLIVEIRA et al., 2012; NOBREGA et al., 2015; NUNES et al., 2016). Já o baixo nível socioeconômico é um fator de risco em consequência do obstáculo ao acesso à saúde, práticas de lazer e suporte social (BORGES et al., 2013; FERREIRA et al., 2015). Ter religião constituise um fator protetor, visto que propicia aos indivíduos enfrentamento das adversidades e esperança para a melhoria da condição de saúde (REIS; MENEZES, 2017). O fato de viver sem companheiro, foi considerado um fator de risco em virtude do fato destes indivíduos estarem mais propensos à solidão (OLIVEIRA et al., 2012; AGUIAR et al., 2014; LOPES et al., 2015).

Na mesma ótica dos fatores de riscos, quanto aos hábitos de vida elencou-se: tabagismo (RAMOS et al., 2015; GULLICH et al., 2016) e não praticar exercício físico (MAGALHAES et al., 2016; HAJJAR et al., 2017). A nicotina altera o funcionamento no sistema neuroendócrino, favorecendo 0 aparecimento e desenvolvimento da psicopatologia (ZAITUNE et al., 2012). A respeito da atividade física, a prática regular de exercício físico atenua o estresse, em virtude da ação das endorfinas. Estes hormônios minimizam o estresse ambiental, desta forma, restringem a manifestação e progressão da sintomatologia depressiva (FERREIRA et al., 2014).

Com referência à condição de saúde, observou-se: avaliação negativa da saúde (BORGES et al., 2013; NOGUEIRA et al., 2014; BRETANHA et al., 2015; RAMOS et al., 2015; GALLI et al., 2016), comorbidades (SOUZA et al., 2013; GALLI 
et al., 2016; HAJJAR et al., 2017), presença de alguma doença crônica (RAMOS et al., 2015; SOUSA et al., 2017), Acidente Vascular Encefálico (AVE) (SILVA et al., 2017) e doenças cardíacas (SILVA et al., 2017), déficit cognitivo (BORGES et al., 2013) incapacidade e dependência funcional (BORGES et al., 2013; BRETANHA et al., 2015), medo e risco de quedas (RAMOS et al., 2015) e ser frágil (RAMOS et al., 2015).

A avaliação negativa da saúde constitui-se um fator de risco dado que o aparecimento de sintomas depressivos interfere negativamente na autoavaliação da saúde (RAMOS et al., 2015; SALERNO et al., 2015). Por outro lado, esta avaliação, pode ser considerada um sintoma (BORGES et al., 2013). Nesta perspectiva, a depressão aumenta a evolução negativa das doenças crônicas e possui alta prevalência depois de um AVE. É responsável ainda por ampliar a incapacidade funcional, pela má qualidade de vida, além de aumentar a taxa de mortalidade (NICCOLI; PARTRIDGE, 2012; AYERBE et al., 2013; LICHTMAN et al., 2014; PARK et al., 2014). Vale realçar que a associação de comorbidades e o uso de polifarmácia, frequentemente observada na população idosa interferem no diagnóstico e no tratamento da depressão (WHO, 2012; BORGES et al., 2013; WHO, 2015).

Sabe-se que o déficit cognitivo é um fator de risco para sintomas depressivos, acredita-se que a depressão seja motivação para a demência ou vice-versa (LIMA et al., 2009). Em contrapartida, sugere-se que o déficit cognitivo melhore, à medida que o paciente inicia e continua a terapêutica para a depressão (BORGES et al., 2013). Em relação a dependência e incapacidade funcional, a perda dessa autonomia, acarreta ao indivíduo o isolamento social, a redução das atividade e apoio social (CHAO, 2014).

Quanto às quedas e auto risco para quedas serem fatores de risco para depressão, isso se dá devido às restrições das atividades por receio de cair, produzem nos idosos alterações psicológicas (DIAS et al., 2011). Tem-se em consideração que a fragilidade foi apontada nesta $\mathrm{Rl}$ como fator de risco para sintomas depressivos, tal relação estaria concatenada ao sedentarismo, astenia e ao esgotamento físico (WOODS et al., 2005; VIEIRA et al., 2013).

No que concerne às queixas dos sintomas depressivos pelos idosos, alguns profissionais de saúde as consideram como consequentes ao processo de envelhecimento. Portanto, a presença destes sintomas pode acarretar a perda da autonomia e agravo dos quadros patológicos preexistentes (OLIVEIRA et al., 2006; WHO, 2015). Comumente a queixa do idoso não se caracteriza somente como física, compreende ainda queixas psíquicas e sociais. Portanto, a avaliação deve ser ampla, com o intuito de detectar alterações presentes, bem como a identificação de sinais que sugerem perda funcional (WHO, 2012; BORGES et al., 2013; WHO, 2015).

Nesta percepção, é imprescindível que os profissionais de saúde, principalmente os atuantes na APS saibam detectar e intervir precocemente no que diz respeito a este agravo (MADEIRA et al., 2013; SOUZA et al., 2013; FENG et al., 2014; NOGUEIRA et al., 2014; NOGUEIRA et al., 2014; BRETANHA et al., 2015; MAGALHAES et al., 2016; SILVA et al., 2017; SOUSA et al., 2017). O rastreamento da depressão em idosos é oportuno a fim de evitar complicações clínicas a curto e a longo prazo (BORGES et al., 2013; BRETANHA et al., 2015; GULLICH et al., 2016). O uso da EDG-15, mesmo preconizado pelo Ministério da Saúde (MS), ainda está em fase inicial (NOGUEIRA et al., 2014). 
No que diz respeito às ações intervencionistas para minimizar o aparecimento e desenvolvimento de sintomas depressivos em idosos, destaca-se que conhecer os fatores de riscos associados a estes sintomas favorece aos profissionais de saúde assegurar o envelhecimento ativo e a promoção da saúde mental da população idosa (BORGES et al., 2013; SOUZA et al., 2013; MADEIRA et al., 2013; BRETANHA et al., 2015; MAGALHAES et al., 2016; GALLI et al., 2016; SOUSA et al., 2017; SILVA et al., 2017).

\section{CONSIDERAÇÕES FINAIS}

Embora o tema desta investigação seja de extrema relevância, existe escassez de produção cientifica. Ainda assim, a EDG-15 é um instrumento empregado em larga escala para avaliação da presença de sintomas depressivos na APS. Diante deste estudo, é fundamental enfatizar que a presença de sintomas depressivos constitui-se uma realidade encontrada em idosos. Os fatores sociodemográficos, condições de saúde, hábitos de vida e auto avaliação negativa da saúde são consideradas variáveis de risco para a presença e desenvolvimento de sintomas depressivos na população idosa.

Por isso, espera-se que a divulgação destas evidências aos gestores e profissionais de saúde gere discussões, transformações e reorganização de suas práticas. Acredita-se que este trabalho poderá subsidiar decisões acerca do investimento público, visando o fortalecimento da APS, com ênfase na saúde mental do idoso.

\section{REFERÊNCIAS}

AGUIAR, A. M. A.; MARQUES, A. P. O.; SILVA, E. C.; COSTA, T. R.; RAMOS, R. S. $P$. S. et al. Prevalência e determinantes de sintomatologia depressiva em idosos assistidos em serviço ambulatorial. Revista Brasileira de Geriatria e Gerontologia, v. 17, n. 4, p. 853-866, 2014. Disponível em: <http://dx.doi.org/10.1590/18099823.2014.13198.>. doi: 10.1590/1809-9823.2014.13198.

ALMEIDA, O. P.; ALMEIDA, A. S. Confiabilidade da versão brasileira da Escala de Depressão em Geriatria (GDS) versão reduzida. Arquivo de Neuropsiquiatria, v. 57, n. 2, p. 421-426, 1999. Disponível em: http://dx.doi.org/10.1590/S0004282X1999000300013>. doi: S0004-282X1999000300013.

ALVARENGA, M. R. M.; OLIVEIRA, M. A. C.; FACCENDA, O. Sintomas depressivos em idosos assistidos pela Estratégia Saúde da Família. Cogitare Enfermagem. v. 15 , n. 2, p. 217- 224, abr.jjun. 2010. Disponível em: <http://dx.doi.org/10.5380/ce.v15i2.17850>. doi: 10.5380/ce.v15i2.17850.

ANDRADE, L. H. S. G.; VIANA, M. C.; SILVEIRA, C. M. Epidemiologia dos transtornos psiquiátricos na mulher. Revista de Psiquiatria Clínica, v. 33, n. 2, p. 43-54, 2006. Disponível em: <http://dx.doi.org/10.1590/S010160832006000200003>. doi: 10.1590/S0101-60832006000200003.

AYERBE, L.; AYIS, S.; WOLFE, C. D.; RUDD, A. G. Natural history, predictors and outcomes of depression after stroke: systematic review and meta-analysis. The British Journal of Psychiatry, v. 202, n. 1, p. 14-21, 2013. Disponível em: <http://dx.doi.org/10.1192/bjp.bp.111.107664>. doi: 10.1192/bjp.bp.111.107664. 
BORGES, L. J.; BENEDETTI, T. R. B.; XAVIER, A. J.; D'ORSI, E. Fatores associados aos sintomas depressivos em idosos: EpiFloripa. Revista de Saúde Pública, v. 47, n. 4, p. 701-710, 2013. Disponível em: <http://dx.doi.org/10.1590/S0034-8910.2013047003844>. doi: S00348910.2013047003844.

BRETANHA, A. F.; FACCHINI, L. A.; NUNES, B. P.; MUNHOZ, T. N.; TOMASI, E. et al. Sintomas depressivos em idosos residentes em áreas de abrangência das Unidades Básicas de Saúde da zona urbana de Bagé, RS. Revista Brasileira de Epidemiologia, v. 18, n. 1, p. 1-12, jan./mar. 2015. Disponível em: < http://dx.doi.org/10.1590/1980-5497201500010001>. doi: 10.1590/19805497201500010001.

CAVALCANTE, F. G.; MINAYO, M. C. S.; MANGAS, R. M. N. Diferentes faces da depressão no suicídio em idosos. Ciência \& Saúde Coletiva, v. 18, n. 10, p. 29852994, 2013. Disponível em: <http://dx.doi.org/10.1590/S1413-81232013001000023>. doi: S1413-81232013001000023.

CHAN, C. L.; WANG, C. W.; HO, A. H.; QU, Z. Y.; WANG, X. Y. et al. Symptoms of posttraumatic stress disorder and depression among bereaved and non-bevereaved survivors following the 2018 Sichuan earthquake. Journal os Anxiety Disorders, V. 26, N. 6, P. 673-679, 2012. Disponível em: <http://dx.doi.org/10.1016/j.janxdis.2012.05.002>. doi: 10.1016/j.janxdis.2012.05.002.

CHAO, S. F.Functional disability and depressive symptoms: longitudinal effects of activity restriction, perceived stress, and social support. Aging \& Mental Health, v. 18, n. 6, p. 767-776, 2014. Disponível em: <http://dx.doi.org/10.1080/13607863.2013.87830>. doi: 10.1080/13607863.2013.87830.

COHEN, R.; PASKULIN, L. M. G.; PRIEB, R. G. G. Prevalência de sintomas depressivos entre idosos em um serviço de emergência. Revista Brasileira de Geriatria e Gerontologia, Rio de Janeiro, v. 18, n. 2, p. 307-317, 2015. Disponível em: <http://dx.doi.org/10.1590/1809-9823.2015.14052>. doi: 10.1590/18099823.2015.14052.

CONFORTIN, S. C.; SCHNEIDER, I. J. C.; ANTES, D. L.; CEMBRANEL, F.; ONO, L. $M$. et al. Condições de vida e saúde de idosos: resultados do estudo de coorte EpiFloripa Idoso. Epidemiologia e Serviços da Saúde, Brasília, v. 26, n. 2, p. 305-317, abr./jun. 2017. Disponível em: <http://dx.doi.org/10.5123/S167949742017000200008>. doi: 10.5123/S1679-49742017000200008.

DIAS, R. C.; FREIRE, M. T. F.; SANTOS, E. G. S.; VIEIRA, R. A.; DIAS, J. M. D. et al. Características associadas à restrição de atividades por medo de cair em idosos comunitários. Revista Brasileira de Fisioterapia. São Carlos, v. 15, n. 5, p. 406413, set./out. 2011. Disponível em: <http:// www.scielo.br/pdf/rbfis/v15n5/pt_a11v15n5.pdf>.

FENG, L.; LI, P.; LU, C.; TANG, W.; MAHAPATRA, T. et al. Burden and Correlates of Geriatric Depression in the Uyghur Elderly Population, Observation from Xinjiang, 
China. PLOS ONE, v. 1, p. 1- 16, dez. 2014. Disponível em: <http://doi.org/10.1371/journal.pone.0114139>. doi: 10.1371/journal.pone.0114139.

FERREIRA, L.; RONCADA, C.; TIGGEMANN, C. L.; DIAS, C. P. Avaliação dos níveis de depressão em idosos praticantes de diferentes exercícios físicos. ConScientiae Saúde, v. 13, n. 3, p. 405-410, 2014. Disponível em:<http://doi.org.10.5585/ConsSaude.v13n3.4839>. 10.5585/ConsSaude.v13n3.4839.

FERREIRA, V. M. P.; SILVA, L. N.; FURUVA, R. K.; SCHIMIDT, A.; ROSSE, L. A. et al. Autocuidado, senso de coerência e depressão em pacientes hospitalizados por insuficiência cardíaca descompensada. Revista da Escola de Enfermagem da USP, v. 49, n.3, p. 388-394, 2015. Disponível em: http://dx.doi.org/10.1590/S0080623420150000300005>. doi: 10.1590/S0080-623420150000300005.

FLUETTI, M. T.; FHON, J. R. S.; OLIVEIRA, A. P.; CHIQUITO, L. M. O.; MARQUES, S. Síndrome da fragilidade em idosos institucionalizados. Revista Brasileira de Geriatria e Gerontologia, Rio de Janeiro, v. 21, n. 1, p. 62-71, 2018. Disponível em: <http://dx.doi.org/10.1590/1981-22562018021.170098>. doi: 198122562018021.170098.

GALLI, R.; MORIGUCHI, H.; BRUSCATO, N. M;; HORTA, R. L.; PATTUSSI, M. P. Active aging is associated with low prevalence of depressive symptoms among Brazilian older adults. Revista Brasileira de Epidemiologia, v. 19, n. 2, p. 307-316, abr./jun. 2016. Disponível em: http://dx.doi.org/10.1590/1980-5497201600020008. doi: 10.1590/1980-5497201600020008.

GALVÃO, C. M.; MENDES, K. D. S.; SILVEIRA, R. C. C. P. Revisão integrativa: método de revisão para sintetizar as evidências disponíveis na literatura. In: BREVIDELLI, M. M.; SERTORIO, S. C. M. TCC Trabalho de Conclusão de Curso: guia prático para docentes e alunos da área da saúde. São Paulo: látria, 4 ed, p.105-126.2010.

GULLICH, I.; DURO, S. M. S.; CESAR, J. A. Depressão entre idosos: um estudo de base populacional no Sul do Brasil. Revista Brasileira de Epidemiologia, v. 19, n. 4, p. 691-701, out./dez. 2016. Disponível em: <http://dx.doi.org/10.1590/19805497201600040001>. doi: 10.1590/1980-5497201600040001.

GUTHS, J. F. S.; JACOB, M. H. V. M.; SANTOS, A. M. P. V.; AROSSI, G. A.; BERIA, J. U. Perfil sociodemográfico, aspectos familiares, percepção de saúde, capacidade funcional e depressão em idosos institucionalizados no Litoral Norte do Rio Grande do Sul, Brasil. Revista Brasileira de Geriatria e Gerontologia, Rio de Janeiro v. 20, n. 2, p. 175-185, 2017. Disponível em: <http://dx.doi.org/10.1590/1981-22562017020.160058>. doi: 10.1590/198122562017020.160058.

HAJJAR, R.; GAUDENCI, G.; GAUDENCI, E. M.; SANTOS, A. S. Depressive symptoms and associated factors in elderly people in the Primary Health Care. Revista da Rede de Enfermagem do Nordeste, v. 18, n. 6, p. 727-733, nov./dez. 2017. Disponível em: <http://dx.doi.org/10.15253/2175-6783.2017000600004>. doi: 10.15253/2175-6783.2017000600004. 
HELLWIG, N.; MUNHOZ, T. N.; TOMASI, E. Sintomas depressivos em idosos: estudo transversal de base populacional. Ciência \& Saúde Coletiva, v. 21, n. 11, p. 3575-3584, 2016. Disponível em: <http://dx.doi.org/10.1590/1413812320152111.19552015>. doi: 10.1590/1413-812320152111.19552015.

HOFFMANN, E. J.; RIBEIRO, F.; FARNESE, J. M.; LIMA, E. W. B. Sintomas depressivos e fatores associados entre idosos residentes em uma comunidade no norte de Minas Gerais, Brasil. Jornal Brasileiro de Psiquiatria, v. 59, n. 3, p. 190197, 2010. Disponível em: <http://www.scielo.br/pdf/jbpsiq/v59n3/a04v59n3.pdf>.

JUNG, J.; ROH, D.; LUA, Y. S.; KIM, D. H. The moderating effect of religion on the relationship between depression and suicidal ideation in the elderly. The Journal of Nervous and Mental Disease, v. 205, n. 8, p. 605-610, ago. 2017. Disponível em: <http://dx.doi.org/10.1097/NMD.0000000000000637>.

doi: 10.1097/NMD.0000000000000637.

KANG, S. Y.; BASHAM, R.; KIM, Y. J. Contributing Factors of Depressive Symptoms Among Elderly Korean Immigrants in Texas. Journal of Gerontological Social Work, v. 56, p. 67-82, 2013. Disponível em: <http://dx.doi.org/10.1080/01634372.2012.734369>. doi: 10.1080/01634372.2012.734369.

KAUP, A. R. BYERS, A. L. FALVEY, C.; SIMONSICK, E. M.; SATTERFIELD, S. et al. Trajectories of Depressive Symptoms in Older Adults and Risk of Dementia. Jama Psychiatry, v. 73, n. 5, p. 525-531, may, 2016. Disponível em: <http://dx.doi.org/10.1001/jamapsychiatry.2016.0004>. doi: 10.1001/jamapsychiatry.2016.0004.

KLEIN, C. H.; BLOCH, K. V. Estudos seccionais. In: MEDRONHO, R. A.; BLOCK, K. V.; LUIZ, R. R.; WERNECK, G. L. Epidemiologia. São Paulo: Atheneu, 2009. p. 193-219.

LICHTMAN, J. H.; FROELICHER, E. S.; BLUMENTHAL, J. A.; CARNEY, R.M.; DOERING, L. V. et al. Depressão como fator de risco para pior prognóstico em pacientes com síndrome coronariana aguda: revisão sistemática e recomendações: uma declaração científica da American Heart Association. Circulation, v. 129, n. 12, p. 1350-1369, mar. 2014. Disponível em: <http://dx.doi.org/10.1161/CIR.0000000000000019>. doi: 10.1161/ CIR.0000000000000019.

LIMA, M. T. R.; SILVA, R. S.; RAMOS, L. R. Fatores associados à sintomatologia depressiva numa coorte urbana de idosos. Jornal Brasileiro de Psiquiatria. v. 58, n. $1, \quad$ p. $1-7, \quad 2009$ Disponível em: < http://www.scielo.br/pdf/ibpsig/v58n1/a01v58n1.pdf>.

LOPES, J. M.; FERNANDES, S. G. G.; DANTAS, F. G.; MEDEIROS, J. L. A. Associação da depressão com as características sociodemográficas, qualidade do sono e hábitos de vida em idosos do Nordeste brasileiro: estudo seccional de base populacional. Revista Brasileira de Geriatria e Gerontologia, Rio de Janeiro, v. 18, 
n. 3, p. 521-531, 2015. Disponível em: <http://dx.doi.org/10.1590/18099823.2015.14081>. doi: 10.1590/1809-9823.2015.14081.

MADEIRA, T. C. S.; AGUIAR, M.I. F.; BERNARDES, A. C. F.; ROLIM, I. L. T. P.; SILVA, R. P. et al. Depressão em idosos hipertensos e diabéticos no contexto da atenção primária em saúde. Revista de Atenção Primária à Saúde, v. 16, n. 4, p. 393-398, out./dez. 2013. Disponível em: <https://aps.ufjf.emnuvens.com.br/aps/article/view/1895/762>.

MAGALHAES, J. M.; CARVALHO, A. M. B.; CARVALHO, S. M.; ALENCAR, D. C.; MOREIRA, W. M. et al. Depressão em idosos na Estratégia Saúde da Família. Revista Mineira de Enfermagem, v. 20, e947, p. 1-6, 2016. Disponível em: Disponível em:<http://dx.doi.org/10.5935/1415-2762.20160016>. doi: 10.5935/14152762.20160016

MELO, B. R. S.; DINIZ, M. A. A.; CASEMIRO, F. G.; FIGUEIREDO, L. C.; ORLANDI, A. A. S. et al. Escola Anna Nery, v. 21, n. 4, p. 1-8, 2017 . Disponível em:<http://dx.doi.org/10.1590/2177-9465-EAN-2016-0388>. doi: 10.1590/2177-9465EAN-2016-0388.

MENDES, K. D. S.; SILVEIRA, R. C. C. P.; GALVAO, C. M. Revisão integrativa: método de pesquisa para a incorporação de evidências na saúde e na enfermagem. Texto \& Contexto Enfermagem, Florianópolis, v. 17, n. 4, p. 758-764, dez. 2008. Disponível em:<http://dx.doi.org/10.1590/S0104-07072008000400018>. doi: 10.1590/S0104- 07072008000400018.

NASCIMENTO, I. M. T.; MELlO, P. F.; COUTO, A. M.; GUIMARAES, G. L.; MENDOZA, I. Y. Q. Associação entre características sociodemográficas e sintomas depressivos em idosos hospitalizados. Revista da Rede de Enfermagem do Nordeste, v. 19, n. 6, p. 749-755, nov./dez. 2017. Disponível em: <http://dx.doi.org/10.15253/2175-6783.2017000600007>. doi: 10.15253/21756783.2017000600007.

NICCOLI, T. PARTRIDGE, L. Ageing as a risk factor for disease. Current Biology, v. 22, n. 7, p. 741-752, 2012. Disponível em: <http://dx.doi.org/10.1016/j.cub.2012.07.024>. doi: 10.1016 / j.cub.2012.07.024.

NOBREGA, I. R. A. P.; LEAL, M. C. C.; MARQUES, A. P. O.; VIEIRA, J. C. M. Fatores associados à depressão em idosos institucionalizados: revisão integrativa. Saúde em Debate, v. 39, n. 105, p. 536-550, 2015. Disponível em: <http://dx.doi.org/10.1590/0103-110420151050002020>. doi: 10.1590/0103110420151050002020.

NOGUEIRA, E. L.; RUBIN, L. L.; GIACOBBOL, S. S.; GOMES, I.; CATALDO NETO, A. Rastreamento dos sintomas depressivos em idosos na Estratégia Saúde da Família, Porto Alegre. Revista de Saúde Pública. v. 48, n. 3, p. 368 - 367, 2014. Disponível em: <http://dx.doi.org/10.1590/S0034-8910.2014048004660>. doi:10.1590/S0034-8910.2014048004660.

NUNES, W. A.; DIAS, F. A.; NASCIMENTO, J. S.; GOMES, N. C.; TAVARES, D. M. S. Avaliação cognitiva e funcional de idosos usuários do serviço público de saúde. 
Escola Anna Nery, v. 17, n. 1, p. 103-111, 2016. Disponível em: <http://dx.doi.org/10.15253/2175-6783.2016000100014>. doi: 10.15253/21756783.2016000100014.

OLIVEIRA, D. A. A. P.; GOMES, L.; OLIVEIRA, R. F. Prevalência de depressão em idosos que frequentam centros de convivência. Revista de Saúde Pública. v. 40, n. 4, p. 734 - 736, 2006. Disponível em: <http://dx.doi.org/10.1590/S0034$89102006000500026>$. doi: S0034-89102006000500026.

OLIVEIRA, M. F.; BEZERRA, V. P.; SILVA, A. O.; ALVES, M. S. C. F.; MOREIRA, M. A. S. P. et al. Sintomatologia de depressão autorreferida por idosos que vivem em comunidade. Ciência \& Saúde Coletiva, v. 17, n.8, p. 2191-2198, 2012. Disponível em: <http://dx.doi.org/10.1590/S1413-81232012000800029 >. doi: 10.1590/S141381232012000800029 .

PARK, M.; KATON, W. J.; WOLF, F. M. Depression and Risk of Mortality in Individuals with Diabetes: A Meta-Analysis and Systematic Review. General Hospital Psychiatry, v. 35, n. 3, p. 217-235, 2013. Disponível em: <http://dx.doi.org/10.1016/j.genhosppsych.2013.01.006>. 10.1016/j.genhosppsych.2013.01.006.

RAMOS, G. C. F.; CARNEIRO, J. A.; BARBOSA, A. T. F.; MENDONÇA, J. M. G; CALDEIRA, A. P. Prevalência de sintomas depressivos e fatores associados em idosos no norte de Minas Gerais: um estudo de base populacional. Jornal Brasileiro de Psiquiatria, v. 64, n. 2, p. 122-131, 2015. Disponível em: <http://dx.doi.org/10.1590/0047-2085000000067>. doi: 10.1590/00472085000000067.

REBello, P. M. P.; LeITE, S. P.; MOUAllemA, A. R. E.; LISBOA, A. C. V.; MARCELINO, A. R. et al. Suspeição de depressão segundo escala geriátrica em uma equipe da Estratégia Saúde da Família. Revista de Atenção Primária a Saúde, v. 14, n. 3, p. 313-318, jul./set. 2011. Disponível em: <https://aps.ufjf.emnuvens.com.br/aps/article/view/1150/509>.

REIS, L. A.; MENEZES, T. M. O. Religiosidade e espiritualidade nas estratégias de resiliência do idoso longevo no cotidiano. Revista Brasileira de Enfermagem, v. 70, n. 4, p. 761-766, 2017. Disponível em: <http://dx.doi.org/10.1590/0034-7167-20160630 >. doi: 10.1590/0034-7167-2016-0630.

RIBEIRO, V. S.; ROSA, R. S.; SANCHES, G. J. C.; RIBEIRO, I. J. S.; CASSOTTI, C. A. Calidad de vida y depresion em idosos en el contexto domiciliar. Revista Electronica Enfermeria Actual em Costa Rica, n. 34, p. 1-14, jan./jun. 2018. Disponível em: <https://doi.org/10.15517/revenf.v0i34.30983>. doi: 10.15517/revenf.v0i34.30983

SALERNO, M. C.; BOLINA, A. F.; DIAS, F. A.; MARTINS, N. P. F.; TAVARES, D. M. S. Autoestima de idosos comunitários e fatores associados: estudo de base populacional. Cogitare Enfermagem, v. 20, n. 4, p. 775-782, out./dez. 2015. Disponível em: <http://dx.doi.org/10.5380/ce.v20i4.41895>. doi: 10.5380/ce.v20i4.41895. 
SANDERS, J. B.; BREMMER, M. A.; COMIJS, H. C.; DEEG, D. J.; BEEKMAN, A. T. Gait Speed and the Natural Course of Depressive Symptoms in Late Life; An Independent Association With Chronicity? Journal of the American Medical Directors Association, v. 17, p. 331-335. 2016. Disponível em:<http://dx.doi.org/10.1016/j.jamda.2015.11.016>. doi: 10.1016/ j.jamda.2015.11.016.

SANTOS, A.; BASTOS, L. L. A. G.; ALEIXO, A. A.; PAULO, T. R. S.; MENDES, E. L. Distribuição, evolução e produção cientifica dos grupos de pesquisa em atividade física e saúde do Brasil. Revista Brasileira de Atividade Física e Saúde, Pelotas, v. $17, \quad$ n. 4 , p. 258-262, ago. 2012. Disponível em: http://seer.uftm.edu.br/revistaeletronica/index.php/aces/article/view/1074/1980>

SIDONE, O. J. G.; HADDAD, E. A.; MENA-CHALCO, J. P. A ciências nas regiões brasileiras: evolução da produção e das redes de colaboração cientifica. TransInformação, v. 28, p. 15-32, 2016. Disponível em:<http://dx.doi.org/ 10.1590/2318-08892016002800002>. doi: 10.1590/2318-08892016002800002.

SILVA, A. R.; SGNAOLIN, V.; NOGUEIRA, E. L.; LOUREIRO, R.; ENGROFF, P. et al. Doenças crônicas não transmissíveis e fatores sociodemográficos associados a sintomas de depressão em idosos. Jornal Brasileiro de Psiquiatria, v. 66, n. 1, p. 45-51, 2017. Disponível em: <http://dx.doi.org/10.1590/0047-2085000000149>. doi: 10.1590/0047-2085000000149.

SILVA, M. T.; GALVAO, T. F.; MARTINS, S. S.; PEREIRA, M. G. Prevalence of depression morbidity among Brazilian adults: a systematic review and meta-analysis. Revista Brasileira de Psiquiatria. v.36, n. 3, p. 262-270, 2014. Disponível em: <http://dx.doi.org/10.1590/1516-4446-2013-1294>. doi: 10.1590/1516-4446-20131294.

SOUSA, K. A.; FREITAS, F. F. Q.; CASTRO, A. P.; OLIVEIRA, C. D. B.; ALMEIDA, A. A. B. et al. Prevalência de sintomas de depressão em idosos assistidos pela Estratégia de Saúde da Família. Revista Mineira de Enfermagem, v. 21, e-1018, p. 1-7, 2017. Disponível em: <http://dx.doi.org/10.5935/1415-2762.20170028>. doi: $10.5935 / 1415-2762.20170028$.

SOUZA, A. S.; SENA, E. L. S.; MEIRA, E. C.; SILVA, D.M.; ALVES, M. S. et al. Perfil sociodemográfico e de saúde de idosos com sintomas depressivos. Revista Enfermagem UERJ, Rio de Janeiro, v. 21, n. 3, p. 355-60, jul./set. 2013. Disponível em: publicacoes.uerj.br/index.php/enfermagemuerj/article/view/7523/5443>. $<$ http://www.e-

SOUZA, M. T.; SILVA, M. D.; CARVALHO, R. Revisão integrativa: o que é e como fazer. Einstein, v. 8, n. 1, p. 102-6, 2010. Disponível em: <http://dx.doi.org/10.1590/s1679-45082010rw1134>. doi: 10.1590/s167945082010 rw1134.

TAVARES, D. M. S.; MATIAS, T. G. C.; FERREIRA, P. C. S.; PEGORARI, M. S.; NASCIMENTO, J. S. et al. Qualidade de vida e autoestima de idosos na 
comunidade. Ciência \& Saúde Coletiva, v. 21, n.11, p. 3557-3564, 2016. Disponível em: <http://dx.doi.org/10.1590/1413-812320152111.03032016>. doi: 10.1590/1413812320152111.03032016 .

TESTON, E. F.; CARREIRA, L.; MARCON, S. S. Sintomas depressivos em idosos: comparação entre residentes em condomínio especifico para idoso e na comunidade. Revista Brasileira de Enfermagem, v. 67, n. 3, p. 450-456, mai./jun. 2014. Disponível em: <http://dx.doi.org/10.5935/0034-7167.20140060>. doi: 10.5935/0034-7167.20140060.

URSI, E. S.; GALVÃO, C. M. Prevenção de lesões de pele no perioperatório: revisão integrativa da literatura. Revista Latino-Americana de Enfermagem, v. 14, n. 1, p. 124-31, jan./fev. 2006. Disponível em: <http://dx.doi.org/10.1590/S010411692006000100017>. doi: 10.1590/S0104-11692006000100017.

VIEIRA, R. A.; GUERRA, R. O.; GIACOMIN, K, C.; VASCONCELOS, K. S. S.; ANDRADE, A. C. S. et al. Prevalência de fragilidade e fatores associados em idosos comunitários de Belo Horizonte, Minas Gerais: dados do Estudo FIBRA. Cadernos de Saúde Pública, v. 29, n. 8, p. 1631-1643, 2013. Disponível em: <http://dx.doi.org/10.1590/0102-311x00126312>. doi: 10.1590/0102-311x00126312.

WOODS, N. F.; LACROIX, A. Z.; GRAY, S. L.; ARAGAKI, A.; COCHRANE, B. B. et al. Frailty: emergence and consequences in women aged 65 and older in the Women's Health Iniciative Observational Study. Journal of the American Geriatrics Society, v. 53, n. $8, \quad$ p. 1321-1330, 2005. Disponível em: <http://dx.doi.org/10.1111/j.1532-5415.2005.53405.x>. doi: 10.1111/j.15325415.2005.53405.x.

WORLD HEALTH ORGANIZATION - WHO. Department of Mental Health and Substance Abuse. Depression: a global public health concern. 2012. Disponível em:

$<$ http://www.who.int/mental_health/management/depression/who_paper_depression_ wfmh_2012.pdf>.

WORLD HEALTH ORGANIZATION - WHO. Mental Heatlh action plan 2013-2020. 2013

Disponível

em: <http://apps.who.int/iris/bitstream/handle/10665/89966/9789241506021_eng.pdf;jses sionid=93F3B912F723ED8341E9438F010FE8C2? sequence $=1$ >.

WORLD HEALTH ORGANIZATION - WHO. World report on ageing and health. $2015 . \quad$ Disponível em: <http://apps.who.int/iris/bitstream/handle/10665/186463/9789240694811_eng.pdf?se quence $=1>$.

WORLD HEALTH ORGANIZATION - WHO. Mental Heatlh Atlas. 2017. Disponível em: $\quad<$ http://apps.who.int/iris/bitstream/handle/10665/272735/9789241514019eng.pdf?ua $=1>$.

ZAITUNE, M. P. A.; BARROS, M. B. A.; LIMA, M. G.F. CESAR, C. L. G.; CARANDINA, L. et al. Fatores associados ao tabagismo em idosos: inquérito de Saúde no Estado de São Paulo (ISA-SP). Cadernos de Saúde Pública, v. 28, n. 4, 
p. 583-595, 2012. Disponível em: <http://dx.doi.org/10.1590/S0102311X2012000300018>. doi: 10.1590/S0102-311X2012000300018. 